\title{
Microscopic structures of Sb-H, Te-H, and Sn-H complexes in silicon
}

\author{
Z. N. Liang \\ Nuclear Solid State Physics, Materials Science Center, Goningen University, Nijenborgh 4, 9747 AG Groningen, The Netherlands \\ P. J. H. Denteneer \\ Instituut-Lorentz, University of Leiden, P.O. Box 9506, 2300 RA Leiden, The Netherlands \\ L. Niesen \\ Nuclear Solid State Physics, Materials Science Center, Goningen University, Nijenborgh 4, 9747 AG Groningen, The Netherlands
}

(Received 10 April 1995)

\begin{abstract}
The microscopic structures of hydrogen-antimony, -tellurium, and -tin complexes in silicon have been studied using first-principles total-energy calculations, in order to obtain a more definitive understanding of the various dopant-hydrogen complexes in $n$-type crystalline silicon. We find that for neutral $\mathrm{SbH}$, $\mathrm{TeH}$, and $\mathrm{SnH}$ complexes, the lowest-energy configurations are similar and of the type AB-Si (the $\mathbf{H}$ is located at the antibonding site of a $\mathrm{Si}$ atom that is adjacent to the impurity). The reaction $\mathrm{SbH}+$ $\mathbf{H} \rightarrow \mathrm{SbH}_{2}$ turns out to be exothermic. The results are consistent with recent experimental results using Mössbauer spectroscopy. For $\mathrm{SbH}_{2}$ various configurations are found that differ only slightly in energy. The lowest-energy configuration of $\mathrm{SbH}_{2}$ complexes exhibits electrical properties similar to those of substitutional $\mathrm{Sb}$. This suggests that the formation of $\mathrm{SbH}_{2}$ not only competes with that of $\mathrm{SbH}$ and $\mathrm{H}_{2}^{*}$, but may also electrically activate the sample.
\end{abstract}

\section{INTRODUCTION}

Hydrogen interacts strongly with dopants in crystalline silicon ( $c-\mathrm{Si})$, often causing a qualitative change in the electrical and optical properties of the dopants. ${ }^{1}$ Because hydrogen is present in practically every step during the processing of Si devices, a substantial body of research has been stimulated to provide an information base for prediction and control. ${ }^{1} \mathrm{H}$ passivation of the shallow dopants is now known to be caused by the formation of neutral dopant-hydrogen complexes. Microscopic structures of the hydrogen-associated complexes are also studied in great depth. The central issue here is a more definitive understanding of the various dopant-hydrogen complexes in $n$-type $c$-Si and their contributions to the electrical properties. One of the important questions is why the passivation efficiency for shallow dopants is generally lower in $n$-type $\mathrm{Si}$ than in $p$-type $\mathrm{Si}$. Furthermore, although a considerable level of understanding of the hydrogen passivation of shallow-level impurities in c-Si has been achieved, little is known about the microscopic nature of the interactions between $\mathbf{H}$ and deep-level impurities (for example, the chalcogens). Experimental evidence has been given for the hydrogen passivation of chalcogen impurities in $c-\mathrm{Si}^{1}{ }^{1}$ However, the passivation mechanism was not clear.

Recently, Mössbauer data on donor-H complexes in $n$ type $c-\mathrm{Si}$ have been presented, which provide evidence for the formation of various donor-H complexes. ${ }^{2,3}$ In these studies, two visible lines and an "invisible fraction" are observed both in $\mathrm{Sb}-\mathrm{H}$ and in $\mathrm{Te}-\mathrm{H}$ systems (the invisible fraction manifests itself as a decrease of the total resonant absorption). The visible lines are attributed to fourfold coordinated substitutional donors and donor-one-H com- plexes ( $\mathrm{SbH}$ and $\mathrm{TeH})$, respectively, whereas the invisible fractions are assumed to be associated with donor-two-H complexes $\left(\mathrm{SbH}_{2}\right.$ and $\left.\mathrm{TeH}_{2}\right)$. Moreover, to account for the observed data in the $\mathrm{Sb}-\mathrm{H}$ system, it is necessary to introduce three species in thermal equilibrium; the wellknown $\mathrm{SbH}$ complex, the $\mathrm{SbH}_{2}$ complex, and a reservoir consisting of $\mathrm{H}$ clustered in the form of $\mathrm{H}_{2}^{*}{ }^{2}$ From a detailed analysis of the time-dependent Mössbauer spectra of the Te-H system it has been suggested that the $\mathbf{H}$ sites in $\mathrm{TeH}$ and $\mathrm{SbH}$ complexes are very similar. ${ }^{3}$

As far as we know, no theoretical calculations have been carried out on $\mathrm{Sb}-\mathrm{H}$ and $\mathrm{Te}-\mathrm{H}$ complexes in $c-\mathrm{Si}$. There are a number of theoretical studies reported on the PH pair in $c$-Si. ${ }^{1}$ While in all cases the results indicate that for a $\mathbf{H}$ atom in the neutral $\mathrm{PH}$ complex the antibonding site of a $\mathrm{Si}$ that is adjacent to the $\mathrm{P}$ atom ( $A B-\mathrm{Si}$ ) is the global energy minimum site for $\mathbf{H}$, there are detailed structural differences, resulting in different calculated $\mathrm{H}$ vibrational frequencies. $A b$ initio Hartree-Fock cluster calculations have been performed on the $\mathbf{P H}_{2}$ system, ${ }^{4}$ suggesting two possible configurations.

In this paper, we focus on the study of the microscopic structures and properties of donor-hydrogen complexes in $c$-Si, using the first-principles pseudopotential-densityfunctional method. In order to make a connection to the Mössbauer experiments, we also investigate the properties of $\mathrm{Sn}-\mathrm{H}$ complexes, because in the Mössbauer experiments ${ }^{2,3}$ one actually measures the $\gamma$ ray emitted from the excited ${ }^{119} \mathrm{Sn}$ nucleus. For neutral $\mathrm{SbH}, \mathrm{TeH}$, and SnH complexes, the lowest-energy configurations are found to be similar and of the type AB-Si (the $\mathbf{H}$ is located at the antibonding site of a Si that is adjacent to the impurity). This is consistent with the Mössbauer results. ${ }^{2,3}$ For the Sb-H system, the binding energies of $\mathbf{H}$ 
atoms in the complexes are calculated. The results indicate that the formation of $\mathrm{SbH}_{2}$ is indeed exothermic, in agreement with the experiment. Furthermore, we find that $\mathrm{SbH}_{2}$ exhibits electrical properties similar to those of substitutional $\mathrm{Sb}$. This suggests that the formation of $\mathrm{SbH}_{2}$ not only competes with that of $\mathrm{SbH}$ and $\mathrm{H}_{2}{ }^{*}$, but may also electrically activate the sample. Microscopic structures of the various impurity-H complexes are proposed. We find a different lowest-energy configuration for $\mathrm{SbH}_{2}$ (and $\mathrm{PH}_{2}$ ) complexes than that proposed by Korpas, Corbett, and Estreicher. ${ }^{4}$

Knowledge about impurity sites and precise positions of the crystal nuclei in the neighborhood of defects in semiconductors is important in order to achieve a better understanding of the impurity electronic structure, ${ }^{5}$ the hyperfine interactions, ${ }^{6}$ diffusion properties, formation and reaction energies, ${ }^{7}$ solubilities, ${ }^{8}$ and the interchange of atoms at heterojunctions. ${ }^{9}$ Therefore, we have also performed calculations for the relaxations of the host crystal around a fourfold-coordinated substitutional (Sn, $\mathrm{Sb}$, and $\mathrm{Te}$ ) impurity in $c$-Si, which is of interest in itself and also helpful for further calculations where the $\mathbf{H}$ atoms are taken into account.

This paper is organized as follows. The calculational details are described in Sec. II. In Sec. III, we present the results for fourfold-coordinated substitutional impurities, i.e., $\mathrm{Sn}, \mathrm{Sb}$, and $\mathrm{Te}$. Then the results for impurity-onehydrogen and impurity-two-hydrogen complexes are given in Sec. IV and Sec. V, respectively. In Sec. VI, we calculate the binding energies of various complexes. In Sec. VII we try to understand the invisible fraction in Mössbauer experiments based on the theoretical results. Conclusions are drawn in Sec. VIII.

\section{CALCULATIONAL DETAILS}

The calculational procedure used in this paper is based on density-functional theory (DFT), using the localdensity approximation (LDA) and ab initio normconserving pseudopotentials. The method is well documented $^{10}$ and has been shown to accurately reproduce and predict ground-state properties of bulk and defective semiconductors, ${ }^{1}$ in particular, for applications to $\mathrm{H}$ and $\mathrm{H}$-impurity complexes in $\mathrm{Si}^{11-13}$ The Hamiltonian in the Kohn-Sham equations for the valence electrons in a crystal is constructed using norm-conserving pseudopo- tentials to describe the interaction between atomic cores (nuclei plus core electrons) and valence electrons. For the exchange and correlation interaction we use the LDA to the exchange and correlation functional that was parametrized by Perdew and Zunger using the Monte Carlo simulations of an electron gas by Ceperley and Alder. ${ }^{10}$

The Kohn-Sham equations are solved by expanding all functions of interest (one-electron wave functions, potentials, etc.) in plane waves and solving the resulting matrix eigenvalue problem. This procedure is iterated until a self-consistent solution is obtained, i.e., until the effective potential for the valence electrons that enters the Hamiltonian equals the effective potential that is calculated from the wave functions that are solutions for this Hamiltonian. From the self-consistent one-electron energies and wave functions the total energy is calculated using a momentum-space formalism. ${ }^{10}$

The properties of different impurities ( $\mathrm{Sb}, \mathrm{Te}$, and $\mathrm{Sn}$ ) and impurity-hydrogen complexes in $\mathrm{Si}$ are studied in a supercell geometry. For every configuration that we consider, the atoms of the host crystal are allowed to relax by minimizing the total energy with respect to the hostcrystal atomic coordinates. Relaxations up to secondnearest neighbors of the impurity and $\mathrm{H}$ atoms are allowed during the calculations.

\section{A. Norm-conserving pseudopotentials}

The Si pseudopotentials are generated according to the Hamann-Schlüter-Chiang scheme, and have been successfully used in previous work. ${ }^{11-13}$ For $\mathrm{Sb}, \mathrm{Al}, \mathrm{Sn}$, and Te we use the tabulated pseudopotential of Bachelet, Hamann, and Schlüter. ${ }^{14}$ No spin-orbit coupling (relativistic effect) is taken into account in these pseudopotentials. For hydrogen we have used the exact $1 / r$ Coulomb potential of the proton. Test calculations were done in Ref. 13 to show that no gain in convergence properties was obtained by using a pseudopotential for hydrogen, and that the $1 / r$ divergence of the Coulomb potential near the core presented no difficulties. ${ }^{13}$

We test these pseudopotentials by calculating the total energies of $\mathrm{Si}, \alpha$-Sn in the diamond structure and of AlSb in the zinc-blende structure as a function of the lattice constants. The calculated values are obtained using a two-atom supercell with the kinetic-energy cutoffs $\left(E_{1}\right.$ and $E_{2}$ ) being 20 and $40 \mathrm{Ry}$ (see Sec. II C for the nota-

TABLE I. Comparison of calculated equilibrium properties with experiments for Si, AlSb, and $\alpha$-Sn. $a_{\text {eq }}$ and $B_{0}$ denote the equilibrium lattice constant and bulk modulus, respectively. The calculated pressure derivative $\left(B^{\prime}\right)$ is also given.

\begin{tabular}{lcccccc}
\hline & \multicolumn{3}{c}{$\mathrm{Si}$} & \multicolumn{2}{c}{ AlSb } & \multicolumn{2}{c}{$\alpha$-Sn } \\
& Calc. & Expt. & Calc. & Expt. & Calc. & Expt. \\
\hline$a_{\text {eq }}(\AA)$ & 5.41 & $5.429(0 \mathrm{~K})^{\mathrm{a}}$ & 6.08 & $6.135(\mathrm{RT})^{\mathrm{b}}$ & 6.39 & $6.483(90 \mathrm{~K})^{\mathrm{c}}$ \\
$B_{0}(\mathrm{Mbar})$ & 0.95 & $0.99^{\mathrm{a}}$ & 0.56 & $0.58^{\mathrm{b}}$ & 0.45 & $0.53^{\mathrm{d}}$ \\
$B^{\prime}$ & 4.45 & & 4.31 & & 4.87 & \\
\hline \hline
\end{tabular}

${ }^{\text {a }}$ Reference 15 .

${ }^{\mathrm{b}}$ Reference 16.

${ }^{\mathrm{c}}$ Reference 17.

${ }^{\mathrm{d}}$ Reference 18. 
tion, $1 \mathrm{Ry}=13.6058 \mathrm{eV}$ ). The results are fitted to Murnaghan's equation of state for solids ${ }^{10}$ to get the equilibrium lattice constant $\left(a_{\text {eq }}\right)$ and bulk modulus $\left(B_{0}\right)$. Table I lists the theoretical and experimental values of $a_{\text {eq }}$ and $B_{0}$. Reasonable agreement has been found between theoretical and experimental results, indicating the reliability of the $\mathrm{Sb}$ and $\mathrm{Sn}$ pseudopotentials for structural studies. However, the results in Table I become slightly worse in going from $\mathrm{Si}$ to AlSb to $\mathrm{Sn}$. This may be related to the fact that the pseudopotentials we use are less suited for heavy elements like $\mathrm{Sn}$, for which spin-orbit coupling may be needed. In all calculations we take 5.41 $\AA$ as the lattice constant for $\mathrm{Si}$ to avoid any spurious stress in the cell, since this lattice constant comes out of calculations of both 2- and 8-atom cells.

\section{B. Supercells}

A supercell is a cell containing a number of primitive unit cells. The primitive unit cell of $\mathrm{Si}$ contains two atoms. As an example, we show in Fig. 1 an 8-atom supercell used in our calculations, which is a cube with the eight atoms numbered (one can easily verify that this cube contains eight atoms). If this 8-atom cell contains one defect, the distance between neighboring defects is $5.41 \AA$ (the lattice constant of $\mathrm{Si}$ ). We also use supercells of 16 and 32 atoms in which defects are separated by 7.65 and $9.37 \AA$.

Typically, the 2-atom unit cell is used to calculate the ground-state properties of $\mathrm{Si}, \mathrm{Sn}$, and AlSb. The 8-atom supercell is employed for preliminary calculations that involve only the first-neighbor relaxations of a defect. Defect levels that show no dispersion for a truly isolated defect do have dispersion when using finite-size supercells. ${ }^{12,13}$ In order to find out the dispersion of the defect level in the band structure it is convenient to use the 16atom cell for comparing the band structures of various complexes, because the 16-atom cell has the same shape of Brillouin zone as the 2-atom cell (face-centered cell in real space). The dispersion of the $\mathrm{H}$-related defect level for $\mathrm{H}$ in $\mathrm{Si}: \mathrm{Sb}$ is found to be about 1.5 and $0.5 \mathrm{eV}$ for the 16- and 32-atom cells, respectively. The final results

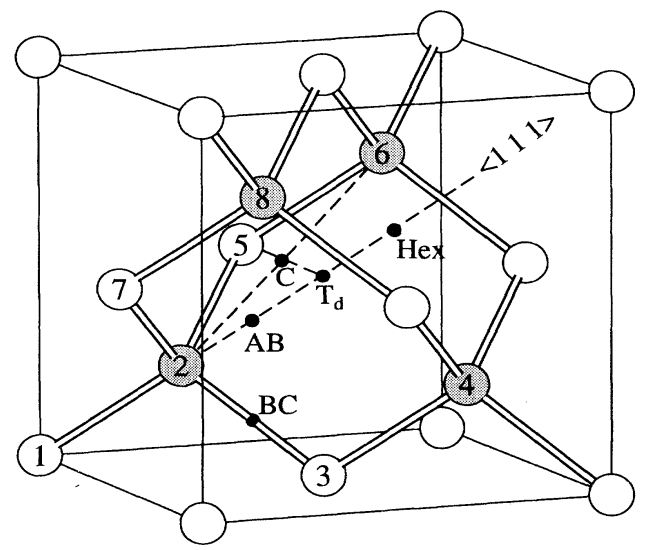

FIG. 1. The 8-atom supercell and names of $\mathbf{H}$ positions in the diamond lattice. The 8 -atom cell is repeated in $\langle 100\rangle$ directions. The four nearest neighbors to a $\mathrm{T}_{d}$ site are shaded. (electronic structures, total energy, total valence charge densities, $\mathbf{H}$ vibrational frequencies, and so on) are obtained using the 32 -atom cell. So unless specified, all results are given for the 32 -atom cells.

\section{Energy cutoffs}

The notation $\left(E_{1} ; E_{2}\right)$ Ry means that plane waves with kinetic energy up to $E_{2} \mathrm{Ry}$ are included in the expansions of wave functions and potentials; waves with kinetic energy up to $E_{1}$ Ry are included in an exact diagonalization of the Hamiltonian matrix, while those between $E_{1}$ and $E_{2} \mathrm{Ry}$ are included in second-order Löwdin perturbation theory; we invariably choose $E_{2}=2 E_{1} \cdot{ }^{12,13}$ For most of the calculations, the energy cutoffs $\left(E_{1}\right.$ and $\left.E_{2}\right)$ are 10 and $20 \mathrm{Ry}$ for 2 - and 8-atom cells and 6 and $12 \mathrm{Ry}$ for 16and 32-atom cells. The choice of supercell size and the corresponding energy cuttoffs has been carefully investigated for $\mathrm{H}$ in $\mathrm{Si}$ and $\mathrm{Si}: \mathrm{B}$ in previous work. ${ }^{12,13}$ The use of the 32-atom cells allows relaxations up to the secondnearest neighbors of the $\mathrm{H}$ atom. The energy cutoffs 6 and $12 \mathrm{Ry}$ are large enough to obtain qualitatively correct energy differences between different positions of the $\mathrm{H}$ atom. For some $\mathrm{H}$ positions of special interest, we use energy cutoffs of 10 and $20 \mathrm{Ry}$ and 32-atom cells to obtain more accurate results.

Figure 2 shows the convergence of ground-state properties of A1Sb and $\alpha-\mathrm{Sn}$, respectively, as a function of kinetic energy cutoff $E_{2}$ in Ry using a 2-atom cell. One sees that these properties are already reasonably converged at an energy cutoff of $12 \mathrm{Ry}$ and fully converged at $20 \mathrm{Ry}$.

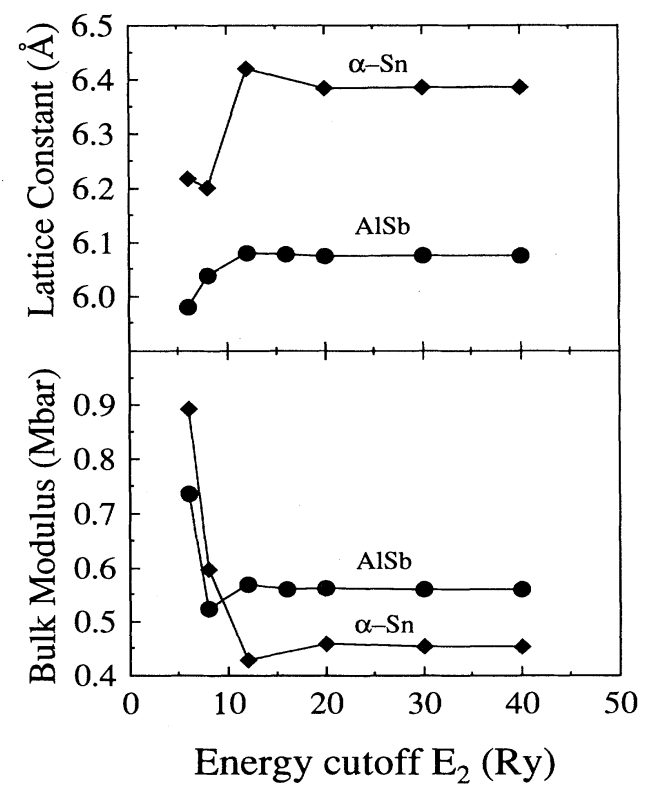

FIG. 2. Convergence of ground-state properties, bulk modulus, and lattice constant of AlSb and $\alpha-\mathrm{Sn}$ as a function of kinetic energy cutoff $E_{2}$ (determining the number of plane waves in the expansion of the wave functions). 


\section{Brillouin-zone integrations}

In two distinct stages of the calculation of the total energy, an integration over the first Brillouin zone has to be performed: (1) calculation of the valence charge density from the one-electron wave functions and (2) calculation of the band-structure energy term from the one-electron energies. $^{12}$ In our calculations integrations over the first Brillouin zone are performed using the special-points scheme. ${ }^{10,19}$ The special points sets are generated with the parameter $q$ equal to 2 for most of the calculations. The number of special points created with $q=2$ depends on the symmetry of the configuration, i.e., the positions of the $\mathbf{H}$ and the impurity atoms. ${ }^{12}$ It is also different for the different supercell sizes that we use. When $\mathbf{H}$ is located at a position on the extension of a Si-impurity bond, $q=2$ results in two special points for the 32-atom cell. For less symmetric $\mathbf{H}$ positions this number can be as high as 4 in the 32 -atom cell.

\section{E. Charge states}

The calculation of charge states requires a careful treatment, since the LDA pseudopotential expressions for the total energy are all derived assuming charge neutrality in the unit cell. Such neutrality is indeed necessary to avoid divergence of the long-range Coulomb terms. We refer to Ref. 13 for a detailed description of the approach for performing the calculations on a charged system. Basically, in this approach one extra electron is taken out or put in the supercell. Then, the neutrality of the system is recovered by assuming that the extra charge is compensated by a charge of opposite sign induced somewhere else in the lattice. In order to avoid the use of very large supercells, the neutrality condition has been satisfied by computing the charge density and the related quantities with the desired number of electrons and by forcing the neutrality condition for the electrostatic contributions (e.g., the Ewald energy). This procedure is justified if the results are shown to be converged as a function of the supercell size.

To avoid confusion, we feel it necessary to make clear the definitions of an electrical level and a defect level in our calculations. An electrical level (or occupancy level) is defined as the position of the Fermi energy for which the ground state switches from $N$ to $N \pm 1$ (for a negative- $U$ center 1 is replaced by 2 ), where $N$ is the number of electrons trapped at the defect labeling the formal charge state of the defect. To give an example: an electrical level $0 /+$ is that position of the Fermi level for which the total energy of the neutral complex equals that of the positively charged complex with the unbound electron at the Fermi level. This definition includes both electronic and lattice readjustment energies and thus is not given by a one-electron eigenvalue calculation. ${ }^{20}$ It is only these levels (characteristic of the boundary between two charge states), and not the one-electron eigenvalues, that can be measured in a quasi-equilibrium experiment. By a "shallow" or a "deep" level we mean a shallow or a deep electrical level in the band gap. In contrast, by a defect level we mean a level induced by the presence of the defect in the band-structure calculation of a one-electron eigenval- ue problem.

Dispersion of the defect levels due to interactions between neighboring supercells places an error bar on the derived position for any defect level. Yet, the main sources of uncertainty in the calculations are the wellknown intrinsic deficiencies of the LDA - in particular, the fact that LDA predicts conduction bands and hence conduction-band-derived energy levels to be too low. However, as pointed out by Van de Walle et al., ${ }^{13}$ a qualitative distinction between various positions of the $\mathbf{H}$ induced level can still be made. Furthermore, while the absolute position of the defect level is uncertain, its relative shift induced by displacements of the impurity or by changes in the charge state is quite reliable. These observations will allow us to derive conclusions about the influence of $\mathbf{H}$ on the electrical properties of impurity-H complexes.

\section{F. Hydrogen vibrational frequencies}

When a minimum-energy configuration is found, we can calculate the hydrogen vibrational frequencies. We move the $\mathbf{H}$ atom away a little from its equilibrium position in directions according to the displacement pattern of the mode of interest (stretching or wagging) and then compute the frequency from the curvature of the energy curve. $^{12}$ Because $\mathbf{H}$ is so much lighter than the other atoms, the positions of these atoms are kept in the minimum-energy configuration. The $\mathbf{H}$ is moved over distances that should be small enough to guarantee the $\mathbf{H}$ is moving in a harmonic potential but large enough to induce total-energy differences beyond the error in the convergence. Typically, the $\mathbf{H}$ atom is moved over distances up to $4 \%$ of a Si-Si bond length, and the induced energy change is up to about $25 \mathrm{meV}$. These energy differences $\Delta E$ are fitted to a parabola $\Delta E=\frac{1}{2} f x_{\mathrm{H}}^{2}$, where $x_{\mathrm{H}}$ is the displacement of the $\mathbf{H}$ atom and $f$ the force constant of the vibrational mode. The frequency $\omega$ can then be obtained by calculating $\omega=\left(f / m_{p}\right)^{1 / 2}$, where $m_{p}$ is the mass of the proton. In this way, vibrational frequencies are calculated with an estimated accuracy of $100 \mathrm{~cm}^{-1}$. We expect to find a higher frequency in going to larger 64-atom cells. ${ }^{21}$

\section{SUBSTITUTIONAL Sn, Sb, AND Te IN $c$-Si}

In Table II we list the calculated results of the bond lengths between $\mathrm{Si}$ and these substitutional impurities in different charge states, which are compared with other theoretical results for Sn (Refs. 9 and 22) and with the experimental data using the extended x-ray absorption fine-structure technique (EXAFS) for $\mathrm{Sb}$ obtained at room temperature for a sample doped with $3.5 \times 10^{18}$ $\mathrm{cm}^{-3} \mathrm{Sb} .{ }^{23}$ No experimental data are available for $\mathrm{Te}$ in Si. In all cases, the nearest-neighboring Si atoms move outward to accommodate the larger impurity atoms, and the second-nearest neighbors also relax outward by 0.04 A.

In $\mathrm{Si}, \mathrm{Sn}$ is an isovalent impurity, while $\mathrm{Sb}$ and $\mathrm{Te}$ are 
TABLE II. The calculated bond lengths (in $\AA$ ) of Si impurity in different charge states, compared with the EXAFS result and other theoretical results. Here the $\mathrm{Si}-\mathrm{Si}$ bond length in $c$-Si is $2.35 \AA$.

\begin{tabular}{lccccc}
\hline \hline \multicolumn{5}{c}{ Charge state } \\
Impurity & $(++)$ & $(+)$ & $(0)$ & Other theory & EXAFS \\
\hline Sn & & & 2.49 & $2.473,{ }^{\mathrm{a}} 2.509^{\mathrm{b}}$ & \\
Sb & & 2.53 & 2.54 & & $2.53 \pm 0.02^{\mathrm{c}}$ \\
$\mathrm{Te}$ & 2.59 & 2.61 & 2.63 & & \\
\hline \hline
\end{tabular}

${ }^{\text {a Reference } 22 .}$

${ }^{b}$ Reference 9.

${ }^{\mathrm{c}}$ Reference 23 .

heterovalent impurities that are, respectively, shallowand deep-level dopants. Isovalent impurities have the same valence electronic structure as the atom they replace. Therefore, they do not change the character of the perfect-crystal chemical bonds. As a consequence, it is possible to calculate the impurity-induced lattice distortions quite accurately, just using information from perfect crystals. ${ }^{9,22,24}$ As exhibited in Table II, our result for Sn agrees very well with the results obtained from simple models, such as a valence force field $\operatorname{model}^{22}$ and a relaxed universal parameter tight-binding theory. ${ }^{9}$

On the other hand, for heterovalent impurities simple models no longer apply. Using state-of-the-art theoretical methods, we obtain results for $\mathrm{Sb}$ that are in very good agreement with the EXAFS data. Note that the local distortion for $\mathrm{Sb}^{0}$ seems to be larger than for $\mathrm{Sb}^{+}$, which may not be expected for a shallow donor like Sb since the hydrogenic state corresponding to such a shallow level is known to extend over several tens of angstroms. This may be due to the finite supercell size effect. To demonstrate this effect we have performed calculations using 8-atom cells for $\mathrm{Sb}$ in neutral and positive charge states. The difference of bond lengths between these two charge states in this case is $0.05 \AA$, considerably larger than $0.01 \AA$ for the 32 -atom cells. This indeed suggests that the charge-state dependence of the local distortions for shallow donors is negligible when the supercell size is large enough.

Chalcogens in $\mathrm{Si}$ are double donors, hence we investigate the charge-state dependence of the lattice relaxations of $\mathrm{Te}$ in all three possible charge states. As shown in Table II, the outwards distortions are larger for the neutral state than for the positively charged states (increasing by about $0.02 \AA$ per electron). Similar behavior has been found for $\mathrm{S}$ in $\mathrm{Si}^{24}$ It is not clear if these small differences are significant; however, the trend can be explained as follows: this charge-state dependence for a chalcogen defect, which possesses a very localized wave function, ${ }^{25}$ is a consequence of the antibonding character of the gap-level wave function, which has a node between the $s$-orbital character at the impurity and the largely $p$ orbital character at the nearest neighbors. ${ }^{24}$ Occupying this wave function with electrons weakens the bonds between the impurity and its nearest neighbors, which implies that the neighbors move away from the impurity. ${ }^{24}$

\section{IMPURITY-ONE-HYDROGEN COMPLEXES IN $c$-Si}

We have performed total-energy calculations for impurity-one-hydrogen complexes. The lowest-energy configuration of $\mathbf{H}$ in different charge states of the complex was determined by examining several interstitial positions for $\mathbf{H}$ as shown in Fig. 1. These include the bondcenter (BC), antibonding (AB), tetrahedral $\left(T_{d}\right)$, hexagonal (Hex), and $C$ sites, where $C$ labels the position at the center of the rhombus formed by the two neighboring Si$\mathrm{Si}$ bonds. There are inequivalent $\mathrm{Hex}$ and $C$ sites in the presence of an impurity, which are denoted by $\mathbf{H e x}^{\prime}$ and $C^{\prime}$. The four nearest neighbors to a $T_{d}$ site are shaded.

\section{A. SbH complexes}

Figure 3 displays three structural models of neutral $\mathrm{SbH}$ complexes [Figs. 3(a) -3(c)] with trigonal symmetry. The energies relative to model (a) are also shown. The numbers in $\AA$ are displacements of the atoms from their perfect Si lattice sites, which are shown with dashed cir-

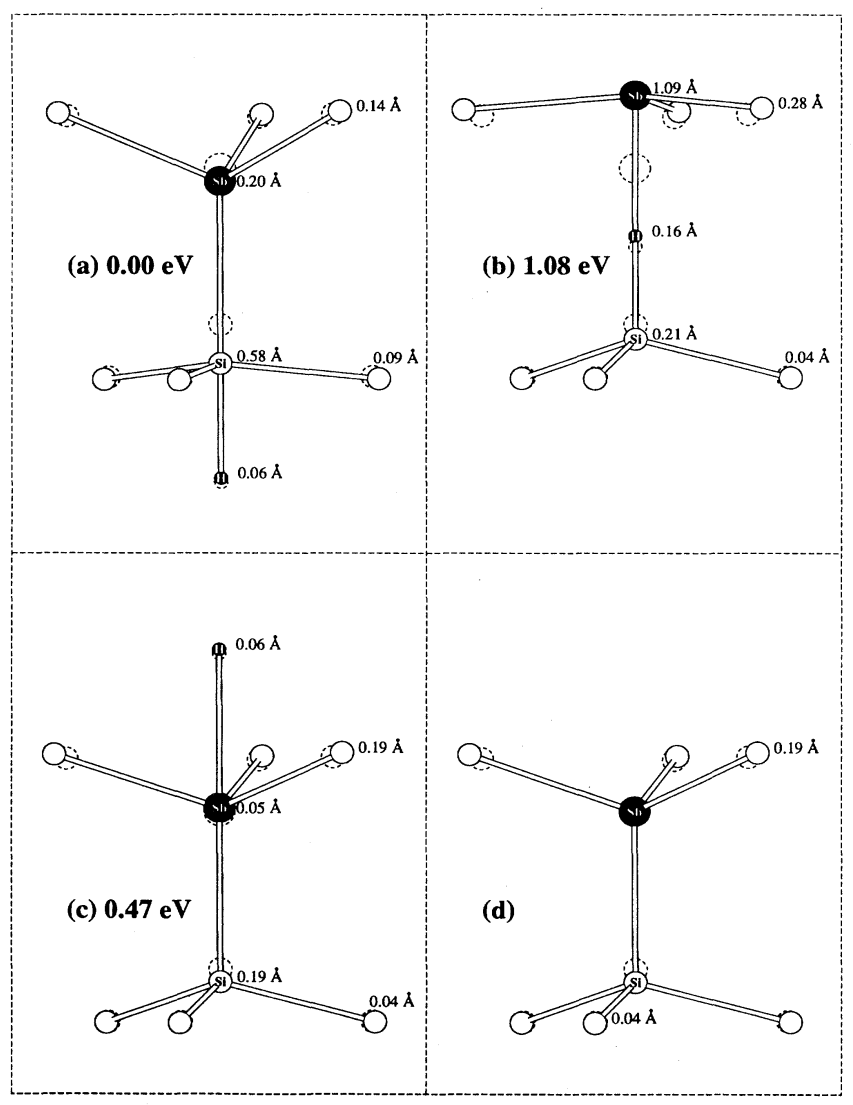

FIG. 3. (a)-(c) Three structural models of neutral SbH complexes in $c$-Si called $\mathrm{AB}-\mathrm{Si}, \mathrm{BC}$, and $\mathrm{AB}-\mathrm{Sb}$ configurations. Their energies (using $20 \mathrm{Ry}$ and 32 -atom cells), relative to model (a), are also shown. For comparison the relaxations around the substitutional $\mathrm{Sb}$ are shown in (d). The numbers in $\AA$ are displacements of the atoms from their perfect $\mathrm{Si}$ lattice sites, which are shown with dashed circles (for $\mathbf{H}$ these sites are perfect $\mathbf{B C}$, $T_{d}$ sites). 
cles (for $\mathrm{H}$ these sites are perfect $\mathrm{BC}$ or $T_{d}$ sites). Moreover, we find that the $C$ and $C^{\prime}$ sites are about 1.9 and 1.5 $\mathrm{eV}$ higher in energy than the lowest-energy configuration [Fig. 3(a)], respectively, and that the Hex and Hex' sites are about $0.7 \mathrm{eV}$ higher in energy and practically do not involve any relaxations of the neighboring atoms. We will not further discuss these sites. For comparison the relaxations around the substitutional $\mathrm{Sb}$ are shown in Fig. $3(\mathrm{~d})$. Figure 4 shows the total valence charge density in
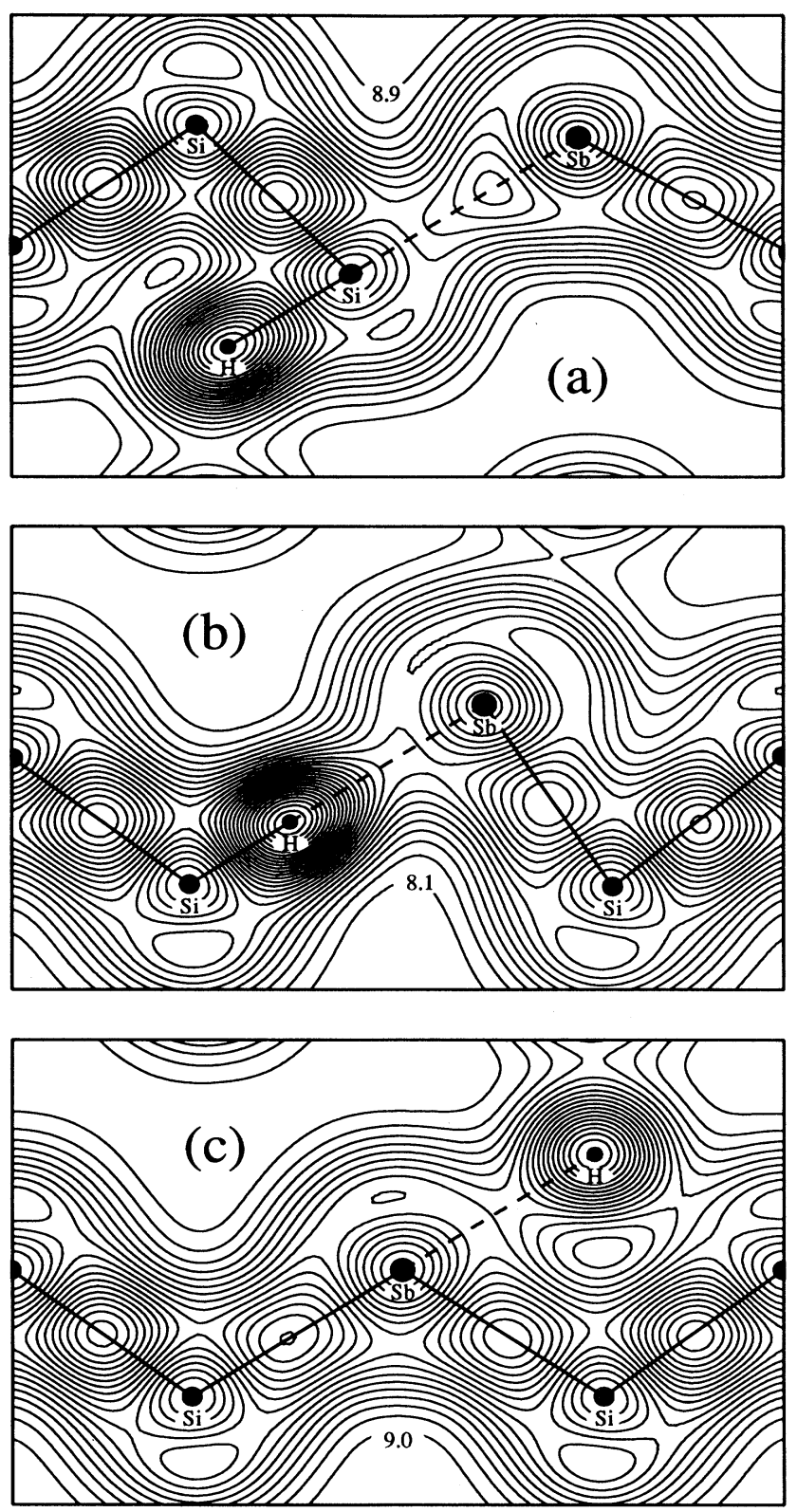

FIG. 4. Total valence charge density in the (110) plane for the three structural models shown in Fig. 3. The black dots indicate atomic positions and the straight lines connect bonded atoms. Dashed lines indicate the broken bonds. The contour spacing is $5.0 e / \Omega$, where $\Omega=160 \AA^{3}$ is the unit volume of the 8 -atom cell (which contains 32 electrons). The lowest-density contour is given for each configuration and the highest-density contour is shown to be around the $\mathrm{H}$ atomic position. the (110) plane for the three configurations displayed in Figs. 3(a) $-3(\mathrm{c})$.

The lowest-energy configuration for the $\mathrm{SbH}$ complexes is the one called AB-Si. The same lowest-energy configuration was also found for $\mathrm{PH}$ and AsH complexes. ${ }^{11,26}$ In this configuration $\mathbf{H}$ is located close to the $T_{d}$ site of a $\mathrm{Si}$ atom bonded to $\mathrm{Sb}$. This $\mathrm{Si}$ atom relaxes towards the $\mathrm{H}$ atom over a large distance of $0.58 \AA$ from its ideal lattice position, and the $\mathrm{Sb}$ atom relaxes by only $0.20 \AA$ in the direction of the $\mathrm{H}$ atom. This results in an effectively broken $\mathrm{Si}-\mathrm{Sb}$ bond and the formation of a $\mathrm{H}-\mathrm{Si}$ bond, as can be seen from Fig. 4(a). Bonding is indicated by the fact that the charge density around the $\mathrm{H}$ atom is clearly modified from the spherical form it has when $\mathbf{H}$ is positioned far away from other atoms; see, for example, Fig. 4(c) where $\mathrm{H}$ is at the AB-Sb site. The H-Si distance is $1.70 \AA$, which is larger than a typical value of about $1.48 \AA$ for a $\mathrm{H}$-Si bond distance in $\mathrm{SiH}_{4}{ }^{27}$ This indicates a weakening of the H-Si bond, which will lead to a lower stretching frequency than for a typical $\mathrm{H}-\mathrm{Si}$ bond [ 2000-2200 cm $\mathrm{cm}^{-1}$ (Ref. 13)]. Indeed, we calculate a stretching frequency of $1393 \mathrm{~cm}^{-1}$, which is, in view of the error bar of $100 \mathrm{~cm}^{-1}$, close to the experimental result of $1562 \mathrm{~cm}^{-1}$. We expect to find a higher frequency in going to 64-atom cells. ${ }^{21}$ Also the calculated frequency of the $\mathrm{H}$ wagging mode of $739 \mathrm{~cm}^{-1}$ is in agreement with the experimental number of $810 \mathrm{~cm}^{-1}$. As will be presented later, other configurations give very different frequencies. The identification of the experimentally observed complex with this AB-Si configuration is strongly supported by the agreement of both calculated frequencies with experiment and the fact that the AB-Si configuration has the lowest energy of all configurations studied. Table III lists the stretching frequencies obtained from infrared measurements in hydrogen passivated $\mathrm{Si}$ doped with $\mathrm{P}, \mathrm{As}$, and $\mathrm{Sb},{ }^{28}$ and from theoretical calculations with the AB-Si model.

Two other local minima configurations shown in Figs. 3(b) and 3(c) are called $\mathrm{BC}$ and AB-Sb configurations. They are, respectively, 1.08 and $0.47 \mathrm{eV}$ higher in energy than configuration (a). We note that for $\mathrm{PH}$ complexes the total-energy differences among these three configurations have been found to be much smaller: they all lie in an energy range of only $0.5 \mathrm{eV} .{ }^{11}$ In the AB-Sb configuration the $\mathrm{H}$ is located nearly at the $T_{d}$ site closest to the $\mathrm{Sb}$ atom, with little relaxation of other atoms. The charge density around $\mathrm{H}$ is shown in Fig. 4(c) to be spherical, indicative of an isolated $\mathrm{H}$ atom (not bonded to other atoms). The stretching vibrational frequency of $\mathbf{H}$ is calculated to be $665 \mathrm{~cm}^{-1}$, which cannot account for the value experimentally observed. The configuration highest in energy of the three shown in Fig. 3 is the one in which $\mathrm{H}$ resides between a $\mathrm{Si}$ and an $\mathrm{Sb}$ atom [Fig. 3(c)], forming a bond with the $\mathrm{Si}$. The bond length of $\mathrm{H}-\mathrm{Si}$ in this configuration is $1.55 \AA$, which is typical for a $\mathrm{Si}-\mathrm{H}$ bond. The $\mathrm{Sb}$ atom relaxes away from the $\mathrm{H}$ atom by $1.09 \AA$ from its ideal position, going through the plane of its nearest three neighboring $\mathrm{Si}$ atoms, which is the consequence of the $\mathrm{H}$ breaking the $\mathrm{Si}-\mathrm{Sb}$ bond and saturating the Si dangling bond. The calculated stretching frequency is $2011 \mathrm{~cm}^{-1}$, much larger than the experimental 
TABLE III. Frequency $\left(\mathrm{cm}^{-1}\right)$ for the $\mathrm{H}$ stretching mode in P-, As-, and Sb-doped $c$-Si. The energy cutoff used in the theoretical calculations is given in parentheses.

\begin{tabular}{|c|c|c|c|c|}
\hline Expt & data $^{a}$ & Zhang and Chadi ${ }^{\mathrm{b}}$ & $\begin{array}{l}\text { Theoretical value } \\
\text { Denteneer et al. }\end{array}$ & This work \\
\hline Si:P & 1555.2 & $1290(11.5 \mathrm{Ry})$ & 1460 (20 Ry) & \\
\hline Si:As & 1561.0 & 1260 (11.5 Ry) & & \\
\hline $\mathrm{Si}: \mathrm{Sb}$ & 1561.7 & & & 1393 (20 Ry) \\
\hline
\end{tabular}

${ }^{\text {a}}$ Reference 28.

${ }^{\mathrm{b}}$ Reference 26.

${ }^{\mathrm{c}}$ Reference 11 .

value (Table III).

Furthermore, we examined for $\mathrm{Sb}$ the other two configurations derived from the $\mathrm{Si}-\mathrm{Sb} \mathrm{BC}$ site, which were viewed as possible metastable configurations by Zhang and Chadi [Figs. 1(d) and 1(e) in Ref. 26]. In one of these configurations the $\mathrm{H}$ atom is at a $\mathrm{BC}$ site with both of its two neighbors relaxing simultaneously away from it, ending up nearly in the plane of their three neighboring atoms. The other configuration is structurally the same as the one shown in Fig. 3(b), yet in this case the Sb and $\mathrm{Si}$ atoms are interchanged (so the $\mathrm{H}$ atom is bonded with $\mathrm{Sb}$ and $\mathrm{Si}$ is threefold coordinated). We find that these two configurations are higher in energy than the BC configuration shown in Fig. 3(b). Moreover, they will change into the $\mathrm{BC}$ configuration without any barrier by basically moving $\mathrm{Si}-\mathrm{H}-\mathrm{Sb}$ as a whole and by simultaneously moving the $\mathrm{Si}$ neighbors of $\mathrm{Sb}$ first out and then in again ("opening and closing the gate"). Therefore, we exclude these two configurations as metastable structures, at least for $\mathrm{Sb}$.

For some configurations the relative stability is also examined with energy cutoffs of 10 and $20 \mathrm{Ry}$, as listed in Table IV. It is demonstrated that the total-energy differences between different $\mathbf{H}$ positions only change about $0.1 \mathrm{eV}$ when increasing energy cutoffs from 6 and $12 \mathrm{Ry}$ to 10 and $20 \mathrm{Ry}$. Denteneer, Van de Walle, and Pantelides $^{12}$ have investigated in detail the energy-cutoff dependence of the calculated energy differences by examining a large set of positions for the $\mathrm{H}$ atom in B-doped Si. They find that it is useful to subdivide the different positions for the $\mathrm{H}$ atom into three regions. In region $\mathrm{I}$ the valence-electron density is very high (e.g., the BC site); in region II the electron density is lower but still considerable (e.g., the $\mathrm{AB}, C$, and $C^{\prime}$ sites); and in region III the electron density is very small $\left(T_{d}\right.$, Hex, and Hex' sites). Regarding convergence with respect to increasing the energy cutoffs, they observed the following: energy differences between sites in the same region change by less than $0.05 \mathrm{eV}$ by going from cutoffs of 6 and $12 \mathrm{Ry}$ to cutoffs of 10 and $20 \mathrm{Ry}$ and therefore may be considered fairly well converged at 6 and 12 Ry. Energy differences between sites in different regions change by about $0.1 \mathrm{eV}$ when the combination of sites is region I and region II. This observation is useful if one wants to extrapolate calculated energy differences to very high-energy cutoffs, which because of computational limitations cannot be handled together with large supercells. When the combination of sites is region I and region III, the energy differences will probably be larger than $0.1 \mathrm{eV}$.

For the positively charged $\mathrm{SbH}$ complex, we note that the AB-Si site is $0.65 \mathrm{eV}$ lower in energy than the $\mathrm{BC}$ site for $\mathbf{H}$ (Table IV), in contrast to the results for $\mathbf{H}$ in positively charged $\mathrm{AsH}$ and $\mathrm{PH}$ obtained from ab initio Hartree-Fock calculations ${ }^{29}$ where the $\mathrm{BC}$ site was found to be lowest in energy. It is not clear whether this disagreement is due to different dopants or different theoretical methods used in the calculations.

Previous studies of $\mathrm{H}$ in $c$-Si (Refs. 12 and 13) have shown that the behavior of a $\mathrm{H}$ atom as an electrically active impurity is intimately tied to the position it assumes in the crystal: the BC (high-charge-density) region giving rise to donor behavior, while the interstitial (low-chargedensity) region is linked to acceptor behavior. Hydrogen behaves as an amphoteric impurity, and it counteracts the electrical activity of dopant impurities in the sample. Whether the impurity is deactivated or activated by $\mathbf{H}$ is merely a consequence of the specific site that $\mathrm{H}$ occupies near the impurity, combined with already present defect levels in the gap. Indeed, for the $\mathrm{SbH}$ pair we find that, similar to the case of the PH pair, ${ }^{11} \mathrm{H}$ is located in a low-density region (AB-Si) and the H-related level is located slightly below the top of the valence band. This

TABLE IV. The energy difference (in eV) with respect to the lowest-energy configuration of the same charge state for $\mathbf{H}$ in the SbH complexes. The energy-cutoff dependence of the calculated energy differences can be seen by comparing the values inside (for $20 \mathrm{Ry}$ ) and outside (for $12 \mathrm{Ry}$ ) the parentheses.

\begin{tabular}{clllllll}
\hline \hline & AB-Si & BC & $\begin{array}{c}\text { H sites } \\
\text { AB-Sb }\end{array}$ & $C$ & $C^{\prime}$ & Hex & Hex' $^{\prime}$ \\
\hline$(+)$ & 0.00 & 0.65 & 0.18 & & & & \\
$(0)$ & $0.00(0.00)$ & $1.17(1.08)$ & $0.45(0.47)$ & 1.9 & 1.5 & 0.7 & 0.7 \\
$(-)$ & 0.00 & 1.17 & 0.50 & & & & \\
\hline \hline
\end{tabular}


level is acceptorlike and becomes occupied by two electrons (one from $\mathrm{H}$ and one surplus electron from $\mathrm{Sb}$ ). The (empty) donor defect level is pushed into the conduction band by the rebonding of the $\mathrm{Sb}-\mathrm{Si}-\mathrm{H}$ complex. ${ }^{30}$ The resulting pair is neutral and electrically inactive.

\section{B. TeH complexes}

For neutral TeH complexes we have also examined the three configurations shown in Figs. 3(a)-3(c). The results show essentially the same behavior as for the case of $\mathrm{SbH}$ complexes. The lowest-energy configuration is of the type AB-Si. In this configuration the $\mathrm{H}$ atom is again found at the $\mathrm{T}_{d}$ site. The $\mathrm{Si}$ atom relaxes towards the $\mathrm{H}$ atom by as much as $0.75 \AA$, ending up in the plane of its three neighboring $\mathrm{Si}$ atoms. The bond length with the $\mathbf{H}$ atom is $1.62 \AA$. The Te atom relaxes by only $0.19 \AA$ in the direction of the $\mathrm{H}$ atom, leading to a Si-Te distance of $2.90 \AA$. The Si-Te bond is effectively broken. The calculated stretching frequency of $\mathrm{H}$ is $1545 \mathrm{~cm}^{-1}$, which is slightly higher than the calculated one for the SbH complex (Table III). This may be merely due to the fact that the Si-H bond length is slightly shorter in the TeH complex than in the SbH. No frequencies from experiment are reported, as far as we know, for the $\mathrm{TeH}$ complexes in Si.

The other two metastable configurations are very similar to the ones shown in Figs. 3(b) and 3(c) but the relaxations of the $\mathrm{Si}$ atoms that are $\mathrm{Te}$ neighbors are larger in this case, simply due to the different relaxations of the $\mathbf{S i}$ atoms that are nearest neighbors of the impurity (see Table II). With respect to $\mathrm{AB}-\mathrm{Si}$, the $\mathrm{BC}$ configuration is $0.97 \mathrm{eV}$ higher and the AB-Te configuration is $0.79 \mathrm{eV}$ higher in energy.

Furthermore, we have calculated the total energy of these three configurations with proper lattice relaxations for the $\mathrm{TeH}$ complexes in the positive charge state. We find that the corresponding structures have few differences between positive and neutral charge states. The implication of this will be discussed in the following section. For the positive charge state, the BC configuration is $1.10 \mathrm{eV}$ higher and the AB-Te configuration is $0.87 \mathrm{eV}$ higher in energy with respect to the AB-Si configuration.

\section{SnH complexes}

In order to compare with Mössbauer experiments, in which the signals from ${ }^{119} \mathrm{SnH}$ complexes were measured, we also performed total-energy calculations for $\mathrm{SnH}$ complexes in three different charge states (positive, neutral, and negative). We find that the AB-Si configuration is the lowest-energy configuration for neutral and negative charge states, whereas the BC configuration is lowest in energy for the positive charge state. Figure 5 shows three configurations of neutral SnH complexes [Figs. 5(a)-5(c)] with the energies relative to the $\mathrm{AB}-\mathrm{Si}$ configuration. Comparing the AB-Si configuration of $\mathrm{SbH}$ and $\mathrm{SnH}$, one sees that $\mathrm{Sn}-\mathrm{Si}-\mathrm{H}$ moves as a whole towards the substitutional site of $\mathrm{Sn}$. Figure 5(d) displays the configuration of fourfold-coordinated substitutional $\mathrm{Sn}$ with relaxations of its first- and second-nearest neighboring Si atoms. Since $\mathrm{Sn}$ is an isovalent impurity in $\mathrm{Si}$, one might expect there is no difference for stable sites of $\mathrm{H}$ in $\mathrm{Si}$ and in $\mathrm{Si}: \mathrm{Sn}$. However, we find that the BC site, a lowest-energy configuration for neutral $\mathbf{H}$ in pure $\mathrm{Si}^{13}$ is no longer a lowest-energy configuration for $\mathbf{H}$ in a neutral $\mathrm{SnH}$ complex (Table V).

The energy differences for $C, C^{\prime}, \mathrm{Hex}$, and $\mathrm{Hex}^{\prime}$ sites with respect to the lowest-energy configurations in different charge states are given in Table $\mathrm{V}$. We note that $\mathrm{H}$ in $\mathrm{SnH}^{+}$as well as in $\mathrm{SnH}^{0}$ complexes has rather lowenergy barriers to move around and that for these two charge states the energy differences between different configurations are small.

Figure 6 gives the $\mathrm{Si}-\mathrm{H}$ distance of the $\mathrm{AB}-\mathrm{Si}$ configurations for $\mathrm{SnH}$ and $\mathrm{SbH}$ complexes as a function of charge state of the complexes. The Si-H distance does not change for the $\mathrm{SbH}$ complexes with the AB-Si configuration, but clearly increases in the $\mathrm{SnH}$ complexes when going from positive to negative charge state. As we mentioned before, the $\mathrm{Si}-\mathrm{H}$ distance in $\mathrm{TeH}$ shows a similar behavior as in $\mathrm{SbH}$. According to the argument presented in Sec. III, the charge-state dependence of the local distortion of $\mathrm{SnH}$ complexes may be the consequence of the antibonding character of the gap-level wave function, indicative of the presence of a $\mathrm{H}$-induced deep level in the gap. In contrast, the charge-state independence of the local distortion of $\mathrm{SbH}$ and $\mathrm{TeH}$ complexes suggests that these systems have no defect levels in the gap.

Figure 7 shows the relative formation energies of $\mathrm{SnH}$ complexes for different charge states, as a function of Fermi-level position. To simplify the plot, we only show the formation energies for the $H$ positions that correspond to the global minimum for a particular charge state, i.e., $\mathrm{BC}$ for $\mathrm{SnH}^{+}$, and $\mathrm{T}_{d}$ for $\mathrm{SnH}^{0}$ and $\mathrm{SnH}^{-}$. Figure 7 (a) shows the values directly obtained from the

TABLE V. The energy difference (in $\mathrm{eV}$ ) with respect to the lowest-energy configuration of the same charge state for $\mathbf{H}$ in the $\mathrm{SnH}$ complexes. The energy-cutoff dependence of the calculated energy differences can be seen by comparing the values inside (for $20 \mathrm{Ry}$ ) and outside (for $12 \mathrm{Ry}$ ) the parentheses.

\begin{tabular}{lccccccr}
\hline \hline & & \multicolumn{7}{c}{ H sites } \\
& Ab-Si & BC & AB-Sn & $C$ & $C^{\prime}$ & Hex & Hex' $^{\prime}$ \\
\hline$(+)$ & $0.29(0.40)$ & $0.00(0.00)$ & $0.51(0.66)$ & 0.32 & 0.04 & 0.35 & 0.38 \\
$(0)$ & $0.00(0.00)$ & $0.30(0.17)$ & $0.22(0.27)$ & 0.70 & 0.36 & 0.24 & 0.23 \\
$(-)$ & $0.00(0.00)$ & $0.87(0.74)$ & $0.17(0.19)$ & 1.40 & 1.00 & 0.51 & 0.47 \\
\hline \hline
\end{tabular}




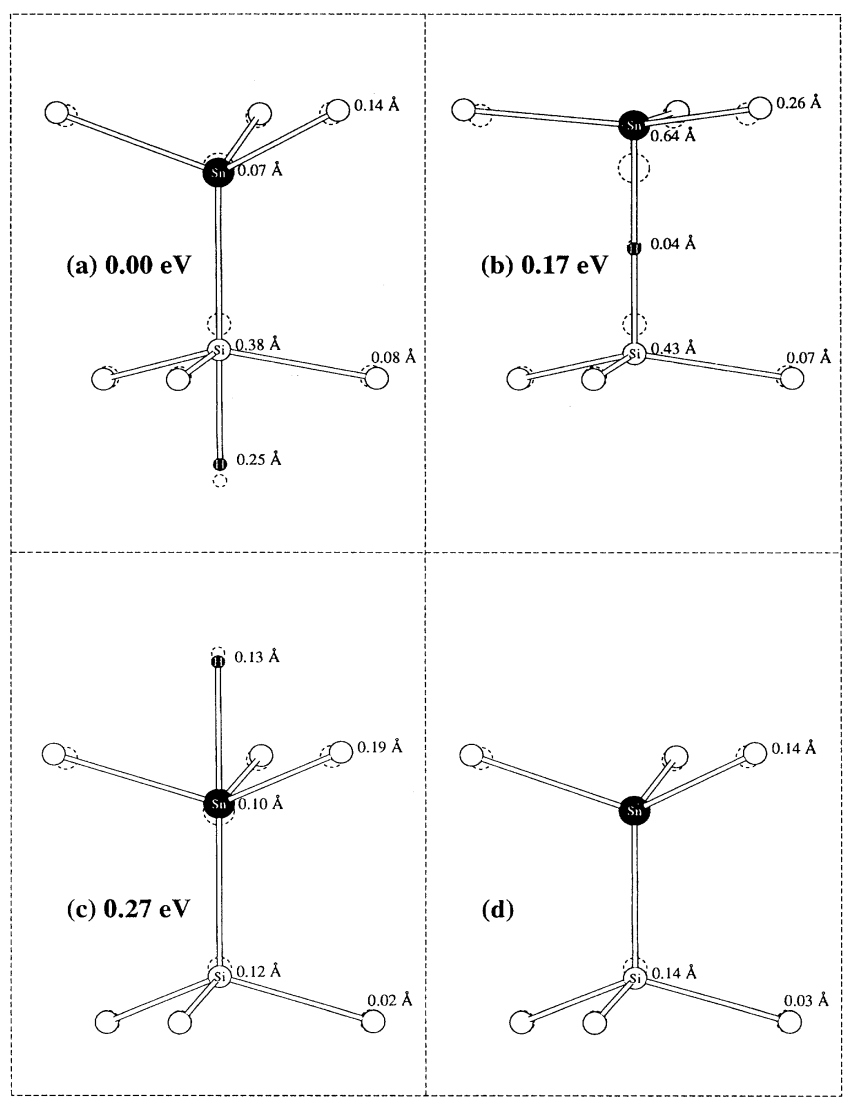

FIG. 5. (a)-(c) Three structural models of neutral SnH complexes in $c$-Si. Their energies (using $20 \mathrm{Ry}$ ), relative to model (a), are also shown. For comparison the relaxations around the substitutional $\mathrm{Sn}$ is shown in (d). The numbers in $\AA$ are displacements of the atoms from their perfect Si lattice sites, which are shown with dashed circles (for $\mathbf{H}$ these sites are perfect $\mathbf{B C}$, $\mathrm{T}_{d}$ sites).

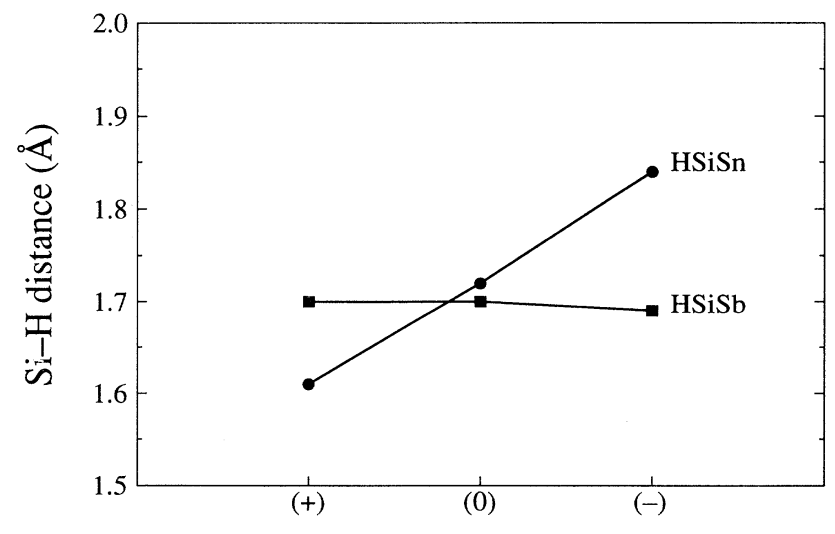

Charge state of the complex

FIG. 6. Si-H distance of the $A B$-Si configurations for $\mathrm{SnH}$ and $\mathrm{SbH}$ complexes as a function of charge state of the complexes.

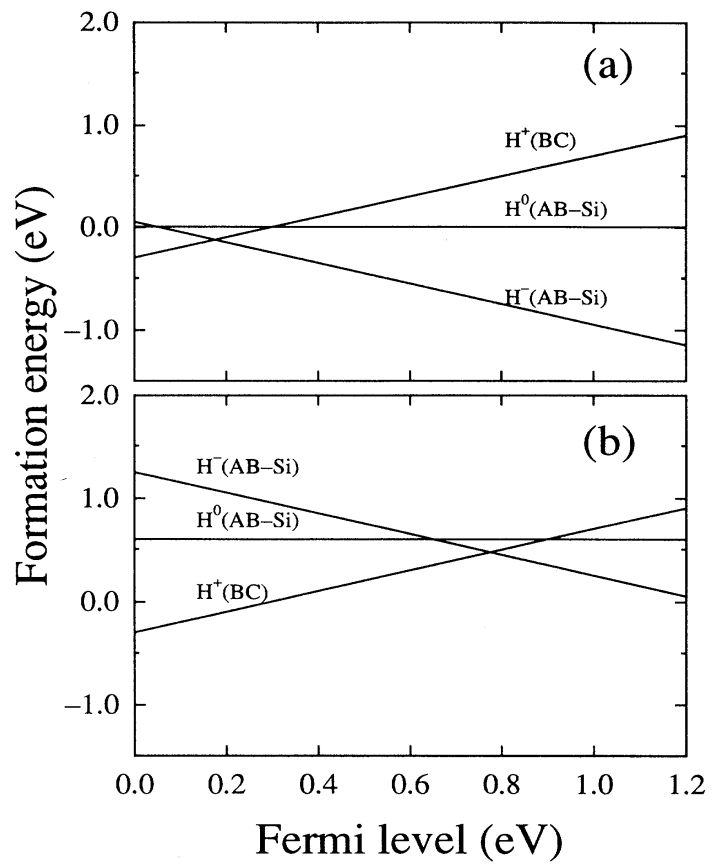

FIG. 7. Relative formation energies for different charge states of the SnH complexes. (a) shows the uncorrected LDA values, while (b) results from applying a simple correction scheme to the energy levels (see text). The zero of energy is arbitrarily chosen as the energy of a neutral $\mathrm{H}$ at the AB-Si site.

LDA calculations. As pointed out in Sec. II E, these suffer from an uncertainty in the position of the defect level. Since the antibonding state is derived primarily from the conduction bands, its defect level position is expected to be similarly underestimated with respect to the valence-band maximum. A correction to the underestimation of the gap will affect, therefore, the total energies for $\mathrm{SnH}^{0}$ and $\mathrm{SnH}^{-}$where the antibonding levels are occupied whereas the energy of $\mathrm{SnH}^{+}$will not change. Figure $7(\mathrm{~b})$ displays the results after the "scissor operation" to bring the band gap into agreement with experiment. ${ }^{13}$ As is shown in Fig. 7, the influence of $\mathrm{H}$ on the electrical activity of the SnH complex depends on the Fermi level position. As in calculations for monatomic $\mathbf{H}$ in $c-\mathrm{Si}^{13}{ }^{13}$ Fig. 7 predicts that the $\mathrm{SnH}$ complex has the negative- $U$ property; it is donorlike in $n$-type $\mathrm{Si}$ and acceptorlike in $p$-type $\mathrm{Si}$. In view of the uncertainty in the LDA energy levels and the use of the scissors operation this prediction is debatable. However, recent experiments have shown that for $\mathbf{H}$ in $c$-Si the theoretical prediction is indeed borne out. ${ }^{39}$ Therefore the present conclusion for $\mathrm{SnH}$ is likely to be correct.

\section{IMPURITY-TWO-HYDROGEN COMPLEXES IN $c$-Si}

\section{A. $\mathrm{SbH}_{2}$ complexes}

Previous Mössbauer results provide evidence for the formation of $\mathrm{SbH}_{2}$ complexes in $n$-type $c$-Si. ${ }^{2}$ However, 
no vibrational frequencies have been reported so far for $\mathrm{H}$ atoms in these complexes. Furthermore, the microscopic structures of these complexes are not well studied. We have explored a number of configurations for neutral $\mathrm{SbH}_{2}$ complexes using an energy cutoff of $20 \mathrm{Ry}$. Figure 8 displays six configurations $[(\mathrm{a})-(\mathrm{f})]$ and their energies relative to (a). The small energy differences leave open the possibility of the occurrence of other configurations. Configuration (a) consists practically of a substitutional $\mathrm{Sb}$ plus a $\mathrm{H}_{2}{ }^{*}$ complex. In the $\mathrm{H}_{2}{ }^{*}$ complex, a $\mathrm{Si}-\mathrm{Si}$ bond is replaced by two $\mathrm{Si}-\mathrm{H}$ bonds, with one $\mathrm{H}$ close to the BC site and the other at an antibonding site. ${ }^{31,32}$ Figure 9 shows the total valence charge density in the (110) plane for configuration (a). From Fig. 8(a) and Fig. 9, it is clearly seen that a $\mathrm{Si}-\mathrm{Si}$ bond is replaced by two $\mathrm{Si}-\mathrm{H}$ bonds. The $\mathrm{H}(\mathrm{BC})$ is only slightly displaced from the perfect $\mathrm{BC}$ site and bonded to the $\mathrm{Si}$ atom that is remote from the $\mathrm{H}\left(T_{d}\right)$, with a bond length of $1.58 \AA$. The $\mathrm{H}\left(T_{d}\right)$ is located practically at the $T_{d}$ site bonded to its nearest $\mathrm{Si}$ atom in this complex with a bond length of $1.60 \AA$. The stretching frequencies are calculated to be $1921 \mathrm{~cm}^{-1}$ for $\mathrm{H}(\mathrm{BC})$ and $1727 \mathrm{~cm}^{-1}$ for $\mathrm{H}\left(T_{d}\right)$. We find that virtually the same values are obtained using an energy cutoff of $12 \mathrm{Ry}$. It is interesting to compare the calculated frequencies for $\mathrm{H}$ atoms in an $\mathrm{SbH}_{2}$ complex with infrared data of $2061.5 \mathrm{~cm}^{-1}$ for $\mathrm{H}(\mathrm{BC})$ and $1838.3 \mathrm{~cm}^{-1}$ for $\mathrm{H}\left(T_{d}\right)$ in an $\mathrm{H}_{2}{ }^{*}$ complex. ${ }^{31}$

In Fig. 8(b) the two $\mathrm{H}$ atoms are located at AB-Si sites, shifted from the $T_{d}$ sites towards the $\mathrm{Sb}$ atom by $0.18 \AA$. (a) $0.00 \mathrm{eV}$

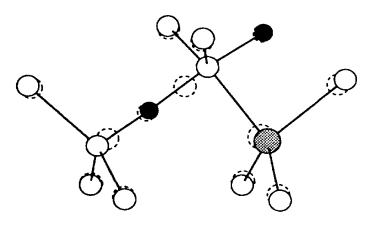

(c) $0.12 \mathrm{eV}$

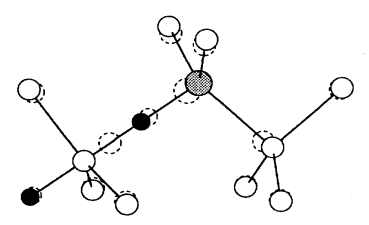

(e) $0.39 \mathrm{eV}$

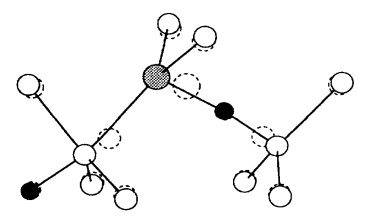

(b) $0.10 \mathrm{eV}$

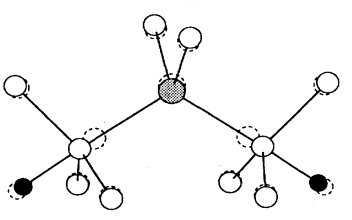

(d) $0.31 \mathrm{eV}$

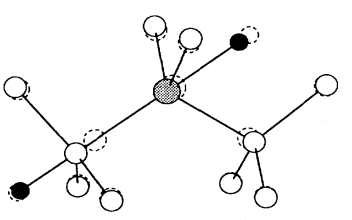

(f) $0.45 \mathrm{eV}$

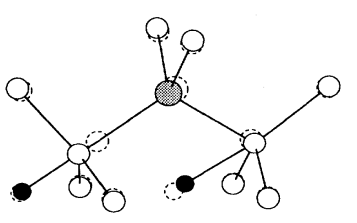

\section{(Si) (Sb)}

FIG. 8. Six structural models for neutral $\mathrm{SbH}_{2}$ complexes in $\mathrm{Si}$. Their energies calculated using a $20 \mathrm{Ry}$ cutoff, with respect to model (a), are also shown. The dashed circles indicate the perfect lattice sites (for $\mathrm{H}$ these are perfect $\mathrm{BC}$ and $T_{d}$ sites).

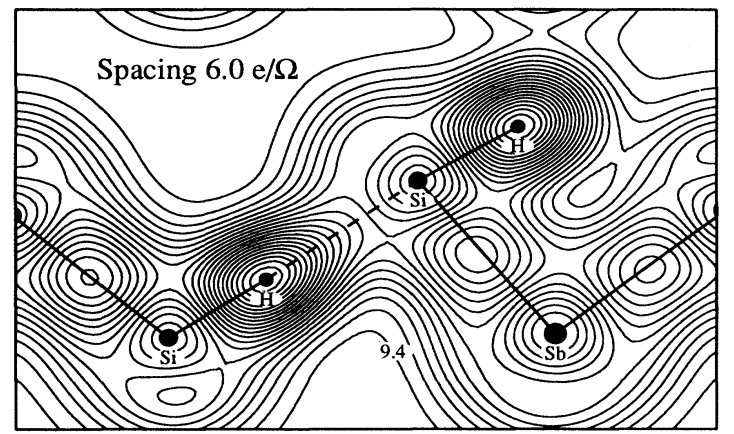

FIG. 9. Total valence charge density in the (110) plane for structural model (a) shown in Fig. 8. The contour spacing is 6.0 $e / \Omega$ (see Fig. 4). It is clearly seen that a $\mathrm{Si}-\mathrm{Si}$ bond is replaced by two H-Si bonds.

The two $\mathrm{Si}$ atoms relax towards the $\mathrm{H}$ atoms by $0.45 \AA$ from their ideal lattice sites, and form bonds with $\mathrm{H}$, with a bond length of $1.71 \AA$. The $\mathrm{Sb}$ atom moves slightly towards the $\mathrm{H}$ atoms symmetrically along a $\langle 100\rangle$ direction. Attempts to break the symmetry by moving the $\mathrm{Sb}$ along a $\langle 111\rangle$ direction towards one of the $\mathrm{H}$ atoms have been found to lead to an increase of the total energy. This configuration is found to be $0.1 \mathrm{eV}$ lower in energy than configuration (a) when using the lower-energy cutoff, reflecting that the convergence of the energy difference between these two configurations is worse $(\sim 0.2 \mathrm{eV})$ than that for many cases of impurity-one-H complexes $(\sim 0.1$ $\mathrm{eV}$ ). The stretching frequency of the $\mathrm{H}$ atoms is calculated to be $1163 \mathrm{~cm}^{-1}$. Configuration (c) has the $\mathrm{Sb}$ and both $\mathrm{H}$ atoms along the same trigonal axis: one $\mathrm{H}$ bridges an $\mathrm{Sb}-\mathrm{Si}$ bond at a near $\mathrm{BC}$ site, the second $\mathrm{H}$ is $\mathrm{AB}$ to that $\mathrm{Si}$ atom. Now the $\mathrm{Si}-\mathrm{H}$ distance is $1.62 \AA$. The calculated stretching frequencies are 1665 and $1801 \mathrm{~cm}^{-1}$ for $\mathrm{H}$ at $\mathrm{AB}-\mathrm{Si}$ and at $\mathrm{BC}$, respectively.

Furthermore, we note that we find configuration (a) to be the lowest-energy configuration also for the $\mathrm{PH}_{2}$ complexes. For $\mathrm{PH}_{2}$ we find that configuration (a) is $0.65 \mathrm{eV}$ lower in energy than (b) and only $0.05 \mathrm{eV}$ lower in energy than (c). Configuration (a) was suggested for P-H complexes in hydrogenated amorphous silicon. ${ }^{33}$ In this configuration, the distances of $\mathrm{P}-\mathrm{H}\left(T_{d}\right)$ and $\mathrm{P}-\mathrm{H}(\mathrm{BC})$ are found to be 2.73 and $2.87 \AA$, respectively, which compare well with the P-H distance of $2.6 \pm 0.3 \AA$ found for half of the $\mathbf{P}$ in hydrogenated amorphous silicon using NMR spin-echo double-resonance spectroscopy. ${ }^{33}$ The other half of the $\mathrm{P}$ in this experiment has a $\mathrm{P}-\mathrm{H}$ distance of $4.1 \pm 0.3 \AA,{ }^{33}$ close to the P-H distance of $4.4 \AA$ calculated for a $\mathrm{PH}$ complex in $c-\mathrm{Si}^{11}$

In previous $a b$ initio Hartree-Fock calculations, ${ }^{4}$ two possible lowest-energy configurations for the $\mathrm{PH}_{2}$ complexes were put forward. The lowest-energy configuration is the one similar to Fig. 8(e) and the second lowest-energy one is similar to Fig. 8(c). We find that (e) is $0.6 \mathrm{eV}$ higher in energy than (a) for $\mathbf{P H}_{2}$. Such a large difference in energy cannot be accounted for by an error in the method. Therefore, we exclude configuration (e) as the global energy minimum configuration for $\mathbf{P H}_{2}$ complexes. 
For the $\mathrm{SbH}_{2}$ complex [Fig. 8(a)] because the symmetry is lower than trigonal, it is no longer easy to identify the defect levels by comparing with complexes with trigonal symmetry. However, based on the arguments in the end of Sec. IV A, we expect the $\mathrm{SbH}_{2}$ complex to have a shallow-donor property. The two $\mathrm{H}$ atoms in the $\mathrm{SbH}_{2}$ form a $\mathrm{H}_{2}{ }^{*}$-like structure which has the electrons of the $H$ atoms being paired with the energies lying in the valence bands, ${ }^{32}$ and therefore, the behavior of the $\mathrm{SbH}_{2}$ complex is largely determined by the behavior of Sb. By inspecting the relative formation energies for different charge states as a function of Fermi level in a way similar to that in Fig. 7 we find that the $\mathrm{SbH}_{2}$ complex indeed shows similar behavior to $\mathrm{Sb}$. Thus, the formation of $\mathrm{SbH}_{2}$ not only competes with that of $\mathrm{SbH}$, but also may depassivate the sample.

\section{B. $\mathrm{SnH}_{2}$ complexes}

We have calculated the total energy for neutral $\mathrm{SnH}_{2}$ complexes with configurations similar to those shown in Fig. 8. The lowest-energy configuration of $\mathrm{SnH}_{2}$ complexes is found to be the one similar to Fig. 8(c). This is not surprising as the same configuration as (c) [and (a) since $\mathrm{Sn}$ is then replaced by $\mathrm{Si}$ ] was found for $\mathrm{H}_{2}{ }^{*}$ in $c$ $\mathrm{Si}^{32}$ Once again, since $\mathrm{Sn}$ is an isovalent impurity in $\mathrm{Si}$, the behavior of $\mathrm{H}$ atoms is the same as if there was no Sn impurity. $\mathrm{H}_{2}{ }^{*}$ is found to be electron-spin-resonance (ESR) inactive, consistent with the experimental failure to detect any ESR signal from $\mathrm{H}$ in $\mathrm{Si}^{32}$ Furthermore, we find that configurations (a) and (b) are 0.1 and $0.7 \mathrm{eV}$ higher in energy, respectively, than configuration (c).

Concerning the electrical properties of the $\mathrm{SnH}_{2}$ complex [Fig. 8(c)], we find no defect level induced in the gap. Furthermore, we find that the resulting complex is neutral and electrically inactive. Again, the $\mathrm{SnH}_{2}$ behaves like $\mathrm{H}_{2}{ }^{*}$.

\section{BINDING ENERGIES OF IMPURITY-H COMPLEXES}

We determine binding energies $E_{b}$ of complexes by calculating the energy difference in the reaction leading to the formation of the complex. When comparing totalenergy differences, one has to make sure that the comparison is made between exactly the same number of atoms of each type. For example, consider the following reaction: $\mathbf{H}^{-}\left(\mathrm{T}_{d}\right)+\mathrm{Sb}^{+} \rightarrow \mathrm{SbH}$. The binding energy of $\mathrm{SbH}$ in this reaction is obtained by calculating the following total energies: (1) $E(\mathrm{SbH})$, the total energy of the neutral $\mathrm{SbH}$ complex as shown in Fig. 3(a); in this case the supercell contains $1 \mathrm{Sb}, 1 \mathrm{H}$, and $31 \mathrm{Si}$ atoms; (2) $E\left(\mathrm{Sb}^{+}\right)$, the total energy of the fully relaxed $\mathrm{Si}$ crystal with a substitutional $\mathrm{Sb}^{+}$; the supercell contains $1 \mathrm{Sb}$ and $31 \mathrm{Si}$ atoms; (3) $E\left(\mathbf{H}^{-}\right)$, the total energy of a fully relaxed Si crystal with a $\mathrm{H}^{-}$at the $\mathrm{T}_{d}$ site; the supercell contains $1 \mathrm{H}$ and $32 \mathrm{Si}$ atoms; (4) $E(\mathrm{Si})$, the total energy of a pure Si crystal; the supercell contains $32 \mathrm{Si}$ atoms. Now, the binding energy of this reaction is given by

$$
E_{b}=-E(\mathrm{SbH})+E\left(\mathrm{Sb}^{+}\right)+E\left(\mathrm{H}^{-}\right)-E(\mathrm{Si}) .
$$

Strictly speaking, one should take into account the contributions from zero-point energies in calculating the
TABLE VI. Calculated binding energies per $\mathbf{H}$ atom in the complexes for some reactions. The energy cutoffs of 20 and 12 $\mathrm{Ry}$ are used in these calculations.

\begin{tabular}{|c|c|c|}
\hline \multirow[b]{2}{*}{ Reactions } & \multicolumn{2}{|c|}{ Binding energy $(\mathrm{eV})$} \\
\hline & $20 \mathrm{Ry}$ & $12 \mathrm{Ry}$ \\
\hline $\mathbf{H}^{0}(\mathrm{BC})+\mathbf{H}^{0}(\mathrm{BC}) \rightarrow \mathrm{H}_{2}^{*}$ & 0.8 & 0.85 \\
\hline $\mathbf{H}^{-}\left(\mathrm{T}_{d}\right)+\mathrm{Sb}^{+} \rightarrow \mathbf{S b H}$ & 0.6 & 0.5 \\
\hline $\mathbf{H}^{0}(\mathbf{B C})+\mathbf{S b} \rightarrow \mathbf{S b H}$ & 1.2 & 1.3 \\
\hline $\mathbf{H}^{0}(\mathbf{B C})+\mathrm{SbH} \rightarrow \mathrm{SbH}_{2}$ & 0.2 & 0.3 \\
\hline $\mathbf{H}^{-}(\mathrm{BC})+\mathbf{P}^{+} \rightarrow \mathbf{P H}$ & & 0.6 \\
\hline $\mathbf{H}^{0}(\mathrm{BC})+\mathbf{P H} \rightarrow \mathrm{PH}_{2}$ & & 0.7 \\
\hline $\mathrm{H}^{0}(\mathrm{BC})+\mathrm{Te} \rightarrow \mathrm{TeH}$ & & 1.4 \\
\hline $\mathbf{H}^{0}(\mathrm{BC})+\mathbf{S n} \rightarrow \mathrm{SnH}$ & & 0.2 \\
\hline $\mathrm{H}^{0}(\mathrm{BC})+\mathrm{SnH} \rightarrow \mathrm{SnH}_{2}$ & & 1.3 \\
\hline
\end{tabular}

binding energies, since the total energies obtained from our calculations are the minima of static parabola curves. However, the zero-point correction has only minor effects on the binding energies we consider here. ${ }^{34}$

In Table VI we present the binding energies for different reactions assumed. In all cases, the binding energies are positive, suggesting that these reactions are exothermic. In particular, the calculations indicate that the formation of $\mathrm{SbH}_{2}$ is indeed possible, in qualitative agreement with the Mössbauer results. ${ }^{2}$ This remains true assuming the reaction $\mathrm{H}^{-}+\mathrm{SbH}^{+} \rightarrow \mathrm{SbH}_{2}$ for the formation of the $\mathrm{SbH}_{2}$ complex, which gives a binding energy of $0.59 \mathrm{eV}$. For $\mathrm{PH}_{2}$ complexes we obtain a value of 0.7 eV compared with $\mathbf{P H}$ and $\mathbf{H}^{0}(\mathrm{BC})$. Furthermore, it is observed that for Sb-H complexes the total-energy differences differ only by a small amount when going from 12- to 20-Ry cutoff, implying that these properties have converged reasonably. Although $\mathrm{Sn}$ is an isovalent impurity in $\mathrm{Si}$, the binding energies of the $\mathbf{H}$ atom(s) in $\mathrm{SnH}$ and $\mathrm{SnH}_{2}$ complexes are positive, indicating that $\mathrm{Sn}$ can also bind $\mathbf{H}$. This also means that the ${ }^{119} \mathrm{Sn}-\mathrm{H}$ complexes resulting from the decay of ${ }^{119} \mathrm{Sb}-\mathrm{H}$ complexes will be stable at low temperatures.

\section{CONSEQUENCES FOR THE MÖSSBAUER EFFECT}

The Mössbauer spectra obtained from $\mathbf{H}$-implanted $\mathrm{Si}: \mathrm{Sb}$ and $\mathrm{Si}: \mathrm{Te}$ samples show a line at $2.34 \mathrm{~mm} / \mathrm{s}$, which is associated with the formation of $\mathrm{SbH}$ and $\mathrm{TeH}$ complexes. Because both ${ }^{119 m} \mathrm{Te}$ and ${ }^{119} \mathrm{Sb}$ will finally decay to ${ }^{119} \mathrm{Sn}$, the fact that the same component is observed for $\mathrm{TeH}$ complexes suggests that in the latter case $\mathbf{H}$ is located at AB-Si, the same site as in $\mathrm{SbH}$ complexes. Our calculations indeed show that in all three cases $\mathbf{S b H}, \mathbf{T e H}$, and $\mathrm{SnH}$ complexes) the energy-minimum configurations are similar (AB-Si). Furthermore, from the calculations we find that the decay of ${ }^{119 m} \mathrm{Te}$ and ${ }^{119} \mathrm{Sb}$ leads to "down hill" relaxations to ${ }^{119} \mathrm{Sn}$; i.e., the newly formed ${ }^{119} \mathrm{Sn}$ atom finds its equilibrium position without surmounting an energy barrier. This relaxation is expected to be much faster than the Mössbauer lifetime ( $25 \mathrm{~ns})$, and therefore we observe the Mössbauer effect from the equilibrium configuration of ${ }^{119} \mathrm{Sn}$.

The situation for the $\mathrm{SbH}_{2}$ complexes is more compli- 
cated. The disappearance of a large part of the Mössbauer absorption after introduction of $\mathbf{H}$ is associated with the formation of $\mathrm{SbH}_{2}$ complexes. ${ }^{2}$ This must be connected with large displacements of the excited ${ }^{119} \mathrm{Sn}$ nucleus during the emission of the Mössbauer $\gamma$ ray. The lower limit of the root-mean-square amplitude of the displacement, $\left\langle x^{2}\right\rangle^{1 / 2}$, is estimated to be $0.15 \AA$, ${ }^{35}$ which cannot be associated with ordinary lattice vibrations. As was pointed out in Ref. 36, a model to explain this phenomenon must contain the following ingredients: (1) $\mathrm{H}$ atoms must be mobile on the time scale of the Mössbauer decay; (2) the motion of the $\mathrm{H}$ atoms must lead to many displacements of the ${ }^{119} \mathrm{Sn}^{*}$ atoms during their whole lifetime. We now discuss these points in view of the microscopic structures of the various complexes presented in this paper.

We have found that there exist configurations with nearly the same energy (differences of about $0.1 \mathrm{eV}$ ) for both $\mathrm{SbH}_{2}$ and $\mathrm{SnH}_{2}$. This may open up the possibility for a $\mathbf{H}$ atom to jump between different configurations, depending on the barrier heights for the $\mathrm{H}$ to migrate from one site to another. At low temperature $\mathrm{H}$ can only move from one position to another by tunneling. Very recently, Cheng and Stavola have shown that the reorientation kinetics of the $\mathrm{BH}$ complex in $\mathrm{Si}$ are nonArrhenius, which suggests that the motion of $H$ in the acceptor-H complexes occurs by thermally assisted tunneling. ${ }^{38}$ However, we think that invoking tunneling does not explain our observations, because in order to get a negligible recoilless fraction, the $\mathrm{H}$ motion must also involve displacements of the heavy $\mathrm{Sn}$ atom, which will drastically slow down the tunneling rate. There have been interesting proposals to explain why $\mathrm{H}$ in some complexes reorients by thermally activated jumps while in others it tunnels rapidly at low temperature. ${ }^{12}$ Watkins ${ }^{37}$ has discussed the qualitative difference between $\mathbf{H}$ motions for defects in which there were substantial relaxations of the H's heavier neighbors ( $\mathrm{Si}$ ) and those that have only small relaxations of the neighbors. It was argued that for the case of small relaxation with a tunneling barrier of $0.5 \mathrm{eV}$, the proton can tunnel at rates of the order of $10^{3} \mathrm{~s}^{-1}$ over a distance of $2.35 \AA$. If there are large relaxations that involve substantial $\mathrm{Si}$ motion when the proton moves, then the appropriate mass for the tunneling species approaches the Si mass, thereby reducing the tunneling rate to about $10^{-41} \mathrm{~s}^{-1}$.

On the other hand, it might be possible that the local temperature around the decaying atom is not so low as the bulk temperature, due to the energy released in the preceding decay. In this case, thermal motion of $\mathbf{H}$ could occur between various configurations with nearly the same energy. At this stage, we have to say that our calculations, although giving a hint for what may happen after the decay from ${ }^{119} \mathrm{Sb}$ to ${ }^{119} \mathrm{Sn}$, do not provide a com- plete explanation for the invisible fraction found in the Mössbauer experiments.

\section{CONCLUSIONS}

We have studied the microscopic structures and the properties of isolated substitutional impurities ( $\mathrm{Sb}, \mathrm{Te}$, and $\mathrm{Sn}$ ) and impurity-H complexes in $c$-Si. For isolated $\mathrm{Sb}$ in $\mathrm{Si}$ we find a nearest-neighbor distance of $2.53 \AA$, corresponding nicely with the EXAFS data. For $\mathrm{Te}$ in $\mathrm{Si}$ the Te-Si distance is somewhat larger than for $\mathrm{Sb}$; it seems to increase in going from the double charged state to the neutral state, which may be due to the localized character of the defect level. For $\mathrm{Sn}^{0}$ we obtained a $\mathrm{Sn}-\mathrm{Si}$ distance of $2.49 \AA$, in good agreement with previous calculations.

The lowest-energy configuration of the $\mathrm{SbH}$ complex has the $\mathrm{H}$ atom close to an AB site of a $\mathrm{Si}$ atom neighboring the $\mathrm{Sb}$ impurity. This $\mathrm{Si}$ atom forms a stronger bond with $\mathrm{H}$ than with the $\mathrm{Sb}$ atom; consequently the $\mathrm{Sb}-\mathrm{Si}$ distance is increased to $2.73 \AA$. The structure resembles that of the PH complex, although the details are different. The resulting configuration is neutral and there is no indication of a defect level in the band gap of $\mathrm{Si}$. This demonstrates the ability of $\mathrm{H}$ to passivate $\mathrm{Sb}$. The calculated vibrational frequencies of $\mathbf{H}$ are in reasonable agreement with experiments. The neutral and positive TeH complexes show a behavior similar to that of the $\mathrm{SbH}$ complex, consistent with experimental results using Mössbauer spectroscopy. For $\mathrm{SnH}$ complexes a different behavior is found. Both a donor and an acceptor level are present, similar to the case of isolated $\mathrm{H}$. The neutral and negative $\mathrm{SnH}$ complexes have structures similar to the $\mathrm{SbH}$ complex, whereas the positive complex has the $\mathrm{H}$ in a $\mathrm{BC}$ site, like in $\mathrm{BH}$.

Calculations on neutral $\mathrm{SbH}_{2}$ complexes yield three configurations that differ less than $0.15 \mathrm{eV}$ in energy. The lowest-energy configuration can be described as a substitutional $\mathrm{Sb}$ atom and an $\mathrm{H}_{2}{ }^{*}$ complex. The second complex has two equivalent $\mathrm{H}$ atoms, each one in the same configuration as in the $\mathrm{SbH}$ complex. The third configuration has axial symmetry and can be described as $\mathrm{Sb}-\mathrm{H}(\mathrm{BC})-\mathrm{Si}-\mathrm{H}\left(T_{d}\right)$. For $\mathrm{PH}_{2}$ complexes similar results have been obtained. In this case the first configuration has clearly the lowest energy. In all cases we find that the reaction $\mathbf{S b H}+\mathbf{H} \rightarrow \mathrm{SbH}_{2}$ is exothermic, in agreement with the interpretation of previous Mössbauer data.

\section{ACKNOWLEDGMENTS}

One of us (Z.N.L.) thanks Professor C. Haas for many valuable discussions. This work is part of the research program of the Foundation for Fundamental Research on Matter (FOM) and was made possible by financial support from the Dutch Organization for the Advancement of Pure Research (NWO).
${ }^{1}$ S. M. Myers et al., Rev. Mod. Phys. 64, 559 (1992); Hydrogen in Semiconductors, edited by J. I. Pankove and N. M. Johnson, Semiconductors and Semimetals Vol. 34 (Academic, New York, 1991); S. Pearton, M. Stavola, and J. W. Corbett, Adv. Mater. 4, 332 (1992).
${ }^{2}$ Z. N. Liang, L. Niesen, and C. Haas, Phys. Rev. Lett. 72, 1846 (1994).

${ }^{3}$ Z. N. Liang and L. Niesen, Phys. Rev. B 51, 11120 (1995).

${ }^{4}$ L. Korpas, J. W. Corbett, and S. K. Estreicher, Phys. Rev. B 46, 12365 (1992). 
${ }^{1}$ S. M. Myers et al., Rev. Mod. Phys. 64, 559 (1992); Hydrogen in Semiconductors, edited by J. I. Pankove and N. M. Johnson, Semiconductors and Semimetals Vol. 34 (Academic, New York, 1991); S. Pearton, M. Stavola, and J. W. Corbett, Adv. Mater. 4, 332 (1992).

${ }^{2}$ Z. N. Liang, L. Niesen, and C. Haas, Phys. Rev. Lett. 72, 1846 (1994).

${ }^{3}$ Z. N. Liang and L. Niesen, Phys. Rev. B 51, 11120 (1995).

${ }^{4}$ L. Korpas, J. W. Corbett, and S. K. Estreicher, Phys. Rev. B 46, 12365 (1992).

${ }^{5}$ M. Scheffler, J. P. Vigneron, and G. B. Bachelet, Phys. Rev. Lett. 49, 1765 (1982).

${ }^{6}$ C. G. Van de Walle, Phys. Rev. Lett. 64, 669 (1990).

${ }^{7}$ C. S. Nichols, C. G. Van de Walle, and S. T. Pantelides, Phys. Rev. Lett. 62, 1049 (1989).

${ }^{8}$ P. A. Fedders and M. W. Muller, J. Phys. Chem. Solids 45, 685 (1984).

${ }^{9}$ E. A. Kraut and W. A. Harrison, J. Vac. Sci. Technol. B 3, 1267 (1985)

${ }^{10}$ P. J. H. Denteneer, Ph.D. thesis, Eindhoven University of Technology, 1987; M. C. Payne, M. P. Teter, D. C. Allan, T. A. Arias, and J. D. Joannopoulos, Rev. Mod. Phys. 64, 1045 (1992); J. Ihm, A. Zunger, and M. L. Cohen, J. Phys. C 12, 4409 (1979); W. Pickett, Comput. Phys. Rep. 9, 115 (1989); J. Perdew and A. Zunger, Phys. Rev. B 23, 5048 (1981); D. M. Ceperley and B. J. Alder, Phys. Rev. Lett. 45, 566 (1980).

${ }^{11}$ P. J. H. Denteneer, C. G. Van de Walle, and S. T. Pantelides, Phys. Rev. B 41, 3885 (1990).

${ }^{12}$ P. J. H. Denteneer, C. G. Van de Walle, and S. T. Pantelides, Phys. Rev. Lett. 62, 1884 (1989); Phys. Rev. B 39, 10809 (1989); 41, 3885 (1990).

${ }^{13}$ C. G. Van de Walle, P. J. H. Denteneer, Y. Bar-Yam, and S. T. Pantelides, Phys. Rev. B 39, 10791 (1989); C. G. Van de Walle, Y. Bar-Yam, and S. T. Pantelides, Phys. Rev. Lett. 60, 2761 (1988).

${ }^{14}$ G. B. Bachelet, D. R. Hamann, and M. Schlüter, Phys. Rev. B 26, 4199 (1982).

${ }^{15}$ M. T. Yin and M. L. Cohen, Phys. Rev. B 26, 5668 (1982).

${ }^{16} \mathrm{~J}$. Baars, P. Glasow, and R. Helbig, Technologie der III-V, IIVI und Nicht-tetraedrisch Gebundene Verbindungen, Landolt Bornstein, New Series, Group III, Vol. 17 (Springer, Berlin, 1984).
${ }^{17}$ D. L. Price and J. M. Rowe, Solid State Commun. 7, 1433 (1969).

${ }^{18}$ C. J. Buchenauer, M. Cardona, and F. H. Pollak, Phys. Rev. B 3, 1243 (1971).

${ }^{19}$ H. J. Monkhorst and J. D. Pack, Phys. Rev. B 13, 5188 (1976).

${ }^{20}$ G. A. Baraff, E. O. Kane, and M. Schlüter, Phys. Rev. B 21, $5662(1980)$.

${ }^{21}$ Y. Zhou, R. Luchsinger, and P. F. Meier, Phys. Rev. B 51, 4166 (1995).

22J. L. Martins and A. Zunger, Phys. Rev. B 30, 6217 (1984).

${ }^{23}$ T. J. van Netten, K. Stapel, and L. Niesen, J. Phys. (Paris), Colloq. 47, C8-1049 (1986).

${ }^{24}$ M. Scheffler, Physica (Amsterdam) 146B, 176 (1987).

${ }^{25}$ J. Bernholc, N. O. Lipari, S. T. Pantelides, and M. Scheffler, Phys. Rev. B 26, 5706 (1982).

${ }^{26}$ S. B. Zhang and D. J. Chadi, Phys. Rev. B 41, 3882 (1990).

${ }^{27}$ CRC Handbook of Chemistry and Physics, edited by R. C. Weast (Chemical Rubber, Boca Raton, FL, 1984).

${ }^{28}$ K. Bergman, M. Stavola, S. J. Pearton, and J. Lopata, Phys. Rev. B 37, 2270 (1988).

${ }^{29}$ S. K. Estreicher, C. H. Seager, and R. A. Anderson, Appl. Phys. Lett. 59, 1773 (1991); S. K. Estreicher and R. Jones, Appl. Phys. Lett. 64, 1670 (1994).

${ }^{30} \mathrm{P}$. J. H. Denteneer, in Proceedings of the 20th International Conference on the Physics of Semiconductors, edited by E. M. Anastassakis and J. D. Joannopoulos (World Scientific, Singapore, 1990), p. 775.

31J. D. Holbech, B. Bech Nielsen, R. Jones, P. Sitch, and S. Öberg, Phys. Rev. Lett. 71, 875 (1993).

${ }^{32}$ K. J. Chang and D. J. Chadi, Phys. Rev. Lett. 62, 937 (1989); Phys. Rev. B 40, 11644 (1989).

33J. B. Boyce and S. E. Ready, Physica (Amsterdam) 170B, 305 (1991); Mater. Sci. Forum 83-87, 1 (1992).

${ }^{34}$ C. G. Van de Walle, Phys. Rev. B 49, 4579 (1994).

${ }^{35}$ Z. N. Liang and L. Niesen, Nucl. Instrum. Methods Phys. Res. Sect. B 63, 147 (1992); Mater. Sci. Forum 83-87, 99 (1992).

${ }^{36}$ Z. N. Liang, Ph.D. thesis, Groningen University, 1994.

${ }^{37}$ G. D. Watkins, Mater. Science Forum 38-41, 39 (1989).

${ }^{38}$ Y. M. Cheng and M. Stavola, Phys. Rev. Lett. 73, 3419 (1994).

${ }^{39}$ N. M. Johnson, C. Herring, and C. G. Van de Walle, Phys. Rev. Lett. 73, 130 (1994). 